\title{
Study on Force Characteristics and Resistance for Water by Amphibious Vehicle
}

\author{
Ling Duan ${ }^{1 \mathrm{a}}$, Kai $\mathrm{YaO}^{2 \mathrm{~b}}$,Xiaochun Pan ${ }^{2 \mathrm{~b}}$,Zhongming Hou ${ }^{2 \mathrm{~b}}$, Xiaowen \\ Zhao $^{2 \mathrm{~b}}$ and Xinmin Tian ${ }^{2 \mathrm{~b}}$ \\ ${ }^{1}$ Chang'an University, Xi'an, China \\ ${ }^{2}$ Xi'an Construction Engineering Research Institution, Xi'an, China

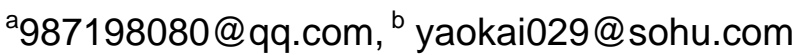

Keywords: Analysis of resistance; water resistance amphibious vehicle;; mechanical characteristics

Abstract. amphibious vehicle for water resistance are complex and difficult to accurately estimate oh, analyzing the force characteristics affecting water sailing by sailing amphibious vehicle posture resistance characteristics of the vehicle. Amphibian Vehicle analyze the composition of the water resistance, friction analysis and calculation methods, shape resistance and wave resistance were studied.

\section{Introduction}

Amphibious vehicle shape changing shape structure, compared with the hull, its water resistance factors more and more computationally complex. Calculation of the amphibious vehicle water features and water resistance force is essential.

\section{Water Characteristics Analysis of Amphibious Vehicle}

\section{Water Stress Analysis of Amphibious Vehicle}

When wheeled amphibious vehicle in water to sail a steady speed, the forces acting on the vehicle, shown in Figure 1.

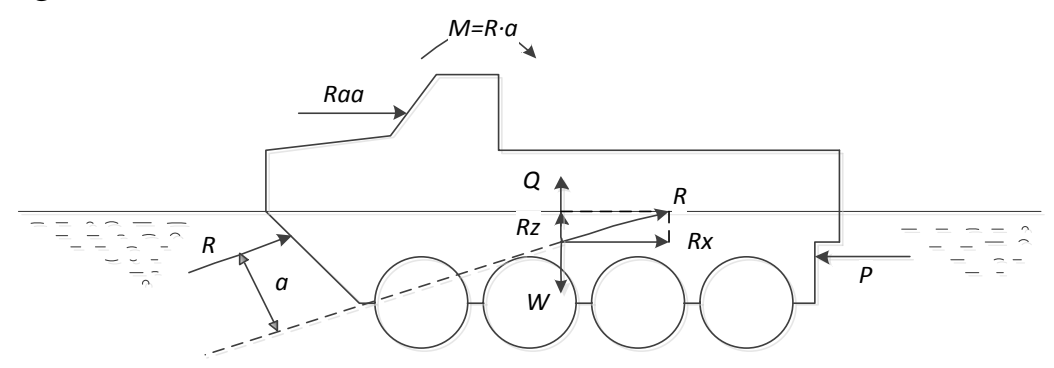

Fig.1 Constant sail the force acting on the vehicle sketch

W- gravity acting on the center of gravity; Q- buoyancy acting on the floating heart; P- propeller thrust;Raa- air resistance, the role of the wind in the central area of the vehicle above the waterline; $\mathrm{R}$ - water resistance

Wheeled amphibious vehicle by the force of the underwater vehicle include:

The force acting on the center of gravity W; floating heart in force Q; propeller thrust P; the role of the vehicle above the waterline in the center of the area upwind air resistance Raa; water resistance $\mathrm{R}$. Among them, the water resistance $\mathrm{R}$ can be decomposed into a horizontal direction backward resistance $\mathrm{Rx}, \mathrm{Rz}$ and lift straight up the vehicle rotation around the center of gravity of the vehicle is formed first or rear tilt moment $\mathrm{M}=\mathrm{R} \cdot \mathrm{a}$. Propulsion is usually applied to the rear of the vehicle, which extend the line through the center of gravity of the vehicle, or easy to form an inclined head and tail. 
Sailing water resistance amphibious vehicle primarily water resistance. Factors affecting the water resistance of the main vehicle speed, body and structure and shape and external conditions part of it.

\section{Navigation State of vehicle impact resistance characteristics analysis}

Water resistance of different types of vehicles with the speed change characteristic that resistance characteristics, can be illustrated by Figure 2 .

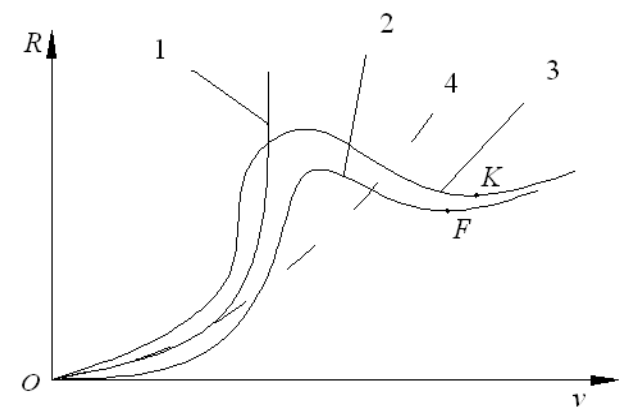

Fig.2 Water resistance curve with the speed

As can be seen from the figure, resistance characteristics of different types of vehicles will be different. According to the vehicle speed volume Froude number FrV, it can be divided into the following three Navigation State:

The first one is drained navigational condition. Because of flight and static state Float changed little, that the equation

$$
W=D=\rho V
$$

D--Displacement; V-- drainage volume; $\rho$-- mass density

Typically unit power $(7.35$ - 18.4) $\mathrm{kW} / \mathrm{t}$ Most of the amphibious vehicle range would fall into that category, its speed is generally low, usually in the (10-15) $\mathrm{km} / \mathrm{h}$, corresponding to the case FrV $<1.0$ in. This thesis light amphibious emergency rescue vehicles fall into this category. Resistance characteristics of this vehicle is shown in the graph in Fig 2.

The second is the transitional sailing condition. Due to the hydrodynamic vehicle speed sailing part of the vehicle is out of the water, this time to satisfy the equation

$$
W=\rho V^{\prime}+R_{z}
$$

$R_{z}$-- lift; $V^{\prime}$ - hydrodynamic volume reduction after displacement

When the speed is in the range $1.0<\mathrm{FrV}<3.0$, Navigation State with more significant changes in speed, while resistance is usually closely related to the state aircraft, shipbuilding industry and therefore the inclusion of such boat speedboat range of areas. However, this type of boat in the coast state compared to hydroplane there are fundamental differences, which is closer to the drainage vessels, so called transitional type of boat, or high-displacement boats.

Drainage type of boat speed boat type features are as follows: slender hull, L / B is relatively large; mostly U-shaped cross section of the hull; from bow to stern, the lateral deadrise angle cross section decreases rapidly, so that tends to zero ; the tail is a square shape. When FrV> 1.0, since these craft would be a serious splash, so that the rapid increase in resistance, therefore, is generally considered the scope of the transitional boats for FrV <1.0. Tests show that the body line of amphibious vehicles modified to streamline or after installation inclusive slide structure can be obtained similar resistance characteristics of high speed displacement boats (Fig2 curve 4).

The third type is coasting condition. Only with a special body shape and high power propulsion of the amphibious vehicle to be able to reach such a state aircraft, vehicles and water sports water slide airfoil vehicles belong to this form. When navigating in this state, the body almost entirely out of the water, $90 \%$ of gravity and hydrodynamic equilibrium, approximately satisfy

$$
W \approx R_{z}
$$


When speed equivalent FrV> 3.0 when the PT boats are all part of a class of hydroplane, this boat is in a coast state, the buoyancy is very small, almost entirely supported by hydrodynamic hull. Amphibious vehicle sliding resistance characteristics shown by the curve 2 . When the vehicle enters the coasting state, the resistance is the lowest point on the curve $2 \mathrm{~F}$, then increase speed, resistance began to rise. This is due to increased friction and splash losses due.

\section{Amphibious vehicle resistance component analysis}

Amphibious vehicle voyage water resistance include form resistance, friction and wave - making resistance of three parts. Amphibious vehicle body shape is essentially different from the ship, its characteristics are: small surface area and length, shape of rapid change, there are a lot of prominent sites, mainly in the action part. Because most of the amphibious vehicle speed is not high, so the sailing resistance amphibious vehicle, friction is relatively small, mainly for form resistance and wave making drag. Experiments have proved that the frictional resistance voyage accounts for about $8 \%$ to $12 \%$ of the total drag, form drag accounted for about $40 \%$ to $80 \%$ of the total resistance (including resistance to the most prominent part of it, of the total resistance of $25 \% \sim 45 \%$ ), wave making resistance and speed are closely related, as the speed in $(9 \sim 11) \mathrm{km} / \mathrm{h}$ in the case, the wave making resistance accounts for about $15 \%$ to $20 \%$ of the total resistance, higher speed, Xing wave resistance will be greater. Only $2 \%$ air resistance can usually be ignored

\section{Analysis of friction}

Frictional resistance acting on the sum of the amphibious vehicle water wet area of all tangential force, with the water flow rate, viscosity and wet area concerned. So far, the ship is still mired in theory is quite flat Froude proposed hull friction and at the same speed, length and wet area of flat friction assumed.

Although in theory the frictional resistance of the surface treatment method are not particularly good, but in his famous series of experiments, the separation surface friction manner deduced following empirical formula, these formulas can be used to predict partially submerged amphibious vehicle body surface friction:

$$
\begin{gathered}
0.242 / C_{r}=\lg \left(v_{0} L / \gamma \times C_{r}\right)=\lg \left(\operatorname{Re} \cdot C_{r}\right) \\
R_{f}=\left(C_{r}+C_{k}\right) \cdot \rho \cdot A v_{0}^{2} / 2
\end{gathered}
$$

$C_{r}$ - Friction coefficient (as a function of Reynolds number); $\gamma$ - Fluid dynamic viscosity; Re-Reynolds $v_{0} L / \gamma ; C_{k}$ - Roughness coefficient (surface roughness of function); $\rho$ - Fluid density; $L$ Vehicle waterline length.

In fresh water in $15{ }^{\circ} \mathrm{C}, \mathrm{Cr}$ and $\mathrm{Ck}$ typical values are shown in Table 1.

Table 1 Cr, Ck coefficients

\begin{tabular}{ccc}
\hline$R e$ & $C_{r}$ & $C_{k}$ \\
\hline $1.5 \times 10^{6}$ & $4.083 \times 10^{-3}$ & $0.4 \times 10^{-3}$ \\
$2.0 \times 10^{6}$ & $3.878 \times 10^{-3}$ & $0.4 \times 10^{-3}$ \\
$2.5 \times 10^{6}$ & $3.719 \times 10^{-3}$ & $0.4 \times 10^{-3}$ \\
$3.0 \times 10^{6}$ & $3.600 \times 10^{-3}$ & $0.4 \times 10^{-3}$ \\
\hline
\end{tabular}




\section{Analysis of shape resistance}

Resistance to size and shape depends on the shape of the object, especially the shape of the tail of the most prominent manifestation of the object. Amphibious vehicle will generally increase the tail length (or to stream segment length), the tail contraction eased, the aspect ratio L / B becomes larger than the width of the draft B / T becomes smaller, making it good rear shape. The shape is actually resistance after immersion in water acting on the front portion of the vehicle body being static pressure and static pressure of the negative forces of the theory, its value

$$
R_{p v}=C_{p v} \frac{1}{2} \rho A v_{0}^{2}
$$

$R_{p v}$-- shape resistance; $C_{p v}$-- shape drag coefficient.

Mill approximate formula given by the Pavlodar available

$$
C_{p v}=0.09 \frac{A_{M}}{A} \sqrt{\sqrt{A_{M}} / 2 \mathrm{~L}_{\mathrm{r}}}
$$

$A_{M}$-- in cross-sectional area; $\mathrm{L}_{\mathrm{r}}$-- to stream length.

The approximate formula described resistance is mainly affected by the shape of the tail shape.

\section{Analysis of wave - making resistance}

Amphibious vehicle to generate waves at the surface navigation, if ignored viscous effect of water, then the resistance is pure wave - making resistance. The study showed that the wave making resistance Rw and water mass density $\rho$, vehicle length $\mathrm{L}$, velocity $\mathrm{v} 0$ and the acceleration of gravity g of the relationship as follows

$$
R_{w}=f\left(\rho \cdot L \cdot v_{0} \cdot g\right)
$$

Dimensional analysis method available

$$
\begin{gathered}
\frac{R_{w}}{\frac{1}{2} \rho A v_{o}^{2}}=f\left(\frac{v_{0}}{\sqrt{g L}}\right) \\
C_{w}=\frac{R_{w}}{\frac{1}{2} \rho A v_{o}^{2}}=f\left(F_{r}\right)
\end{gathered}
$$

Although the expression (10) can not directly calculate the wave making resistance of the actual vehicle, but to the obvious physical revelation, that when speed increased wave making resistance is bound to increase the amphibious vehicle. AD688965 report that, after repeated experiments show that the vehicle, is proportional to the fourth power of the wave making resistance drain amphibious vehicle about the speed of. Figure 3 is a test for a variety of amphibious vehicles and ship models and in kind derived curve. Although the resistance value is relative to the total resistance is concerned, but due to the large proportion of total wave making resistance, studies have shown that increasing the speed of vehicles, almost always appeared a resistance "wall" which way "wall" is 4 the results caused power of relationship.

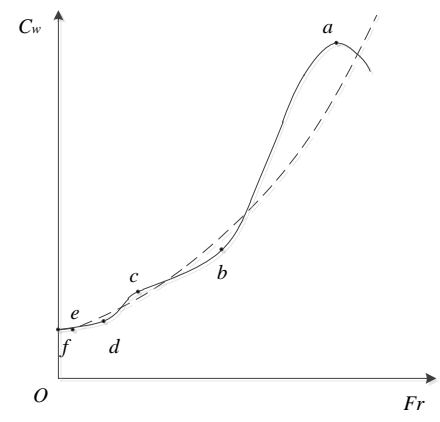

Fig.3 Wave making drag coefficient curve 


\section{Conclusions}

Analysis of the force characteristics affecting water sailing by sailing amphibious vehicle posture resistance characteristics of the vehicle. Amphibian Vehicle analyze the composition of the water resistance, friction analysis and calculation methods, shape resistance and wave resistance were studied.

\section{References}

[1] ZHANG Zhi-rong,ZHAO feng,LI Bai-qi.Numerical calculateions of viscous free-surface flow about ship hull[J].J.of Ship Mechanics.2002,6(6):10-17.

[2] Kim Y H,Choi Y,Jansson C,Larsson L.Linear and nonlinear calculation of the free surface potential flow around ships in shallow water[A].Proceedings,20th ONR Symposium on Naval Hydrodynamics[C],1994.

[3] GAO Qiu-xin,SHU Lei.Numerical simulation of free surface flow around frigate model 5415[J].J.of Ship Mechanics.2002,6(6):1-9.

[4] Miao Q M,Chwang A TThe effect of submerged vertical walls on ship waves[A].The 14th Engineering Mechanics Conference[C],EM2000(CD-Rom),Austin,Texas,USA,2000.

[5] Miyata H,Kanai A.Numerical analysis of structure of free-surface shock wave about a wedge model[J].Journal of Ship Research,Dec.1996,40(4):278-287. 